\title{
A miniaturized device for the measurement of sheet-metal formability using digital image correlation
}

M. E. Leonard, F. Ugo, M. Stout, and J. W. Signorelli

Citation: Review of Scientific Instruments 89, 085114 (2018); doi: 10.1063/1.5042053

View online: https://doi.org/10.1063/1.5042053

View Table of Contents: http://aip.scitation.org/toc/rsi/89/8

Published by the American Institute of Physics

\section{Articles you may be interested in}

A comparison based digital waveform generator for high resolution duty cycle

Review of Scientific Instruments 89, 084101 (2018); 10.1063/1.5004798

Measurement of anisotropic thermal conductivity of a dense forest of nanowires using the $3 \omega$ method Review of Scientific Instruments 89, 084902 (2018); 10.1063/1.5025319

New design techniques of resistive bolometer system for analysis of total radiated power

Review of Scientific Instruments 89, 083501 (2018); 10.1063/1.5026927

Analyzing the audio signals of degenerative arthritis with an electronic stethoscope

Review of Scientific Instruments 89, 085111 (2018); 10.1063/1.5018006

Note: Precision balance for sub-miliNewton resolution direct thrust measurement

Review of Scientific Instruments 89, 086108 (2018); 10.1063/1.5045855

Measurement of the Hall effect at nanoscale with three probes

Review of Scientific Instruments 89, 083904 (2018); 10.1063/1.5030872

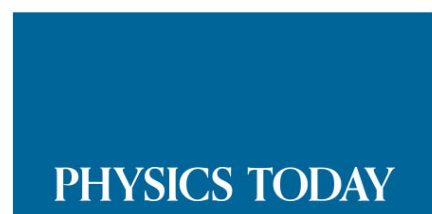

MANAGER'S GUIDE

WHITEPAPERS
Accelerate R\&D with

Multiphysics Simulation

\section{READ NOW}

PRESENTED BY

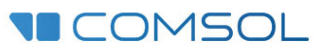




\title{
A miniaturized device for the measurement of sheet-metal formability using digital image correlation
}

\author{
M. E. Leonard, F. Ugo, M. Stout, and J. W. Signorelli \\ Instituto de Física Rosario, Consejo Nacional de Investigaciones Científicas y Técnicas, Universidad Nacional \\ de Rosario (IFIR-CONICET/UNR), 27 de Febrero 210 Bis, 2000 Rosario, Santa Fe, Argentina
}

(Received 29 May 2018; accepted 29 July 2018; published online 22 August 2018)

\begin{abstract}
A laboratory-scale device to obtain forming-limit diagram data was designed to utilize the Marciniak and Kuczynski (MK) sample geometry. The design uses a high-resolution photographic camera, automatic trigger, and light-emitting diode (LED) lighting to record the time history of deformation calculated with the digital-image correlation technique. Because the testing device was miniaturized, it was possible to halt the forming experiments at intermediate strains and recrystallize the MK carrier blank. This permits large formability strains to be obtained without cracks developing at the carrier blank's central hole, an advantage over full-size specimens and conventional testing rates. A number of initial experiments were performed on a zinc alloy sheet $(\mathrm{Zn}-\mathrm{Cu}-\mathrm{Ti})$ over the entire forming-limit range $\left(-0.5 \leq \varepsilon_{2} / \varepsilon_{1} \leq 1\right)$, and the strain fields reduced employing the Bragard criterion to obtain limit strains. These results are compared favorably to previous data of this material obtained with a hemispherical, Nakazima, punch and a circle-grid pattern. Published by AIP Publishing. https://doi.org/10.1063/1.5042053
\end{abstract}

\section{INTRODUCTION}

A metal sheet's ability to deform without the development of fracture or flow localization, during various industrial forming processes such as drawing or stretching, is referred to as the sheet's formability. ${ }^{1,2}$ This characteristic has typically been quantified graphically in in-plane, principal-strain space with a forming limit diagram (FLD). The FLD was originally developed by Keeler ${ }^{3}$ and Goodwin ${ }^{4}$ based on Gensamer's analysis. ${ }^{5}$ For a particular strain state (ratio $\varepsilon_{2} / \varepsilon_{1}=\rho$ ), the limit strain is defined as the strain value where the sheet's deformation ceases to be uniform and a local plastic-strain instability, a neck, begins to develop. To assess the FLD, deformation experiments are conducted over different strain paths (from uniaxial tension, $\rho=-0.5$, to equibiaxial stretching, $\rho=1$ ) and the strain at which a flow instability develops is measured and plotted. A curve is then fit through these points. Strain values above the curve are considered to be unsafe for a forming process, while a processing strain below the curve is safe.

The measurement of the FLD has been standardized, most recently in the norm ISO $12004-2,{ }^{6}$ for sheet samples on the length scale of shop-floor processes (diameters greater than $100 \mathrm{~mm}$ ). This norm specifies two types of testing techniques, which are depicted schematically in Fig. 1 and discussed below.

Nakazima's technique ${ }^{7}$ is the most commonly used one today. Basically, disk-shaped samples are clamped in a die and deformed with a hemispherical shaped punch. Through cutouts on the sides of these disks, giving them an hourglass shape, and different lubrications between the punch and the sample, all strain states are easily attainable. A classic summary of the relationship between the matrix and punch geometries, sample shapes, and lubrication was given by Hecker. ${ }^{8}$ While it is relatively easy to obtain various stress states with this sample geometry, the disadvantage is that the deformations are three dimensional and out-of-plane. This produces large deformation gradients and a through-thickness stress component, albeit small.

The Marciniak and Kuczynski ${ }^{9}$ (MK) experiment is the second technique for determining the forming-limit strains detailed in ISO 12004-2. ${ }^{6}$ In this experiment, the sample sheet and an intermediate carrier blank are fixed in the die and stretched over a planar surface punch. The carrier blank has a central hole, and the friction between the carrier blank and the sample-as the carrier blank expands stretching over the punch-loads the sample. Having the intermediate carrier blank between the punch and the sample prevents tearing of the sample at the punch radius. As is the case for Nakazima's test, different sample and carrier blank widths provide different strain states. For the MK experiment to be successful, having correct dimensions and ratios between the punch, the punch radius, the die diameter and the carrier blank and sample sheet thicknesses is critical. These dimensions are now provided in the ISO-norm. The principal advantage of the MK test is that the sample deformation is planar and the loading stresses are purely biaxial, owing to the expanding hole in the carrier blank that provides a contact-free zone in the sample's center. A disadvantage of the test is that the carrier blank must sustain large deformations without developing radial cracks at the edge of the central hole. Any cracking provides a trigger for the development of a plastic instability in the test piece and invalidates the experiment. ${ }^{6}$ The main advantages and disadvantages of these two tests are summarized in Fig. 1.

Historically, deformations and limit strains in FLD samples were measured through circular or rectangular grids of a known dimension deposited on the specimen's sheet surface. ${ }^{1-9}$ After the experiment, circles or squares of uniform and localized deformation were identified and measured optically with a traveling microscope. This gave safe and insecure strains directly for a particular strain state. A second technique for 


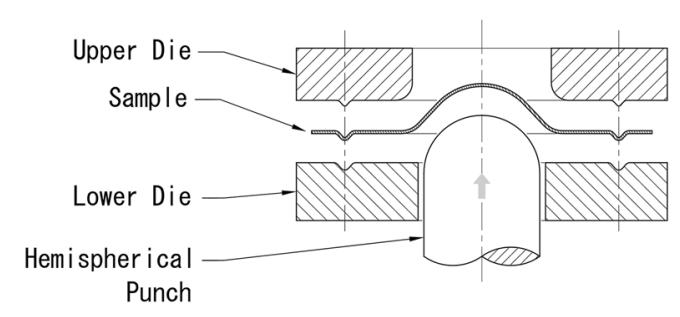

$\begin{array}{ll}\checkmark \text { Allows all strain paths } & \times \text { Nonuniform strain state } \\ \checkmark \text { Simple sample preparation } & \times \text { Thickness stress } \sigma_{3} \neq 0 \\ \checkmark \text { Widely used historically } & \times \text { Requires 3D-DIC program }\end{array}$

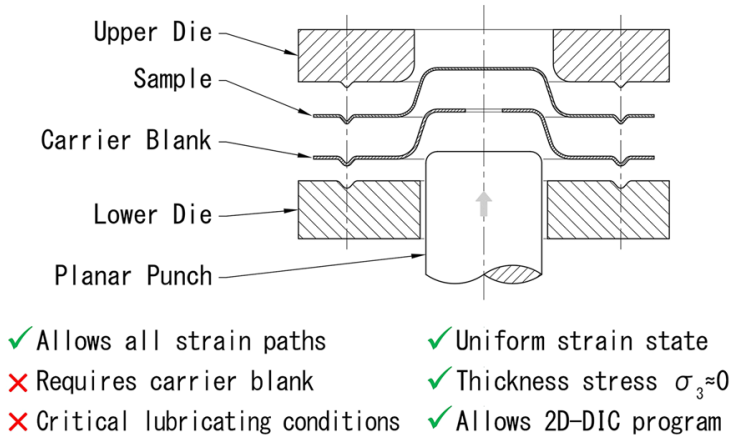

FIG. 1. Sketches and advantages and disadvantages of the two typical tests used to assess FLDs, as discussed in the ISO 12004-2 norm. Upper half: Nakazima's technique; lower half: Marciniak-Kuczynski (MK) test.

determination of the limit strains was developed by Bragard. ${ }^{10}$ In this case, the strains are measured along a line traversing at $90^{\circ}$ to the failure instability or fracture. Grid areas containing either a crack or deformation localization are discounted. These data are then fitted by a polynomial whose value at the center of the instability or fracture is the limit strain. Most recently, the technique of digital image correlation ${ }^{11,12}$ has been applied to measure limit strains. Digital image correlation gives not only full-field deformation data but much greater resolution than the traditional grid technique. For image correlation, a random pattern of fine dark spots is applied over a uniform white background, i.e., a speckle pattern. As deformation of the sample proceeds, the successive images of the speckle pattern are recorded and distortions, expansions, and contractions in the pattern give deformations and subsequently strains through commercial (e.g., GOM) or open source (Ncorr) digital image correlation programs. ${ }^{11,13,14}$ If the Nakazima sample is used, a commercial code is required because these can handle three-dimensional deformations. The MK experiment is planar, and the open-source, two-dimensional code Ncorr is adequate for image analysis. No matter which correlation program is used, limit strains can be calculated using either the Bragard technique ${ }^{10}$ or the time history of deformation..$^{15}$

We designed equipment for measuring limit strains using the MK sample technique because of its two-dimensional nature and the ability to use the two-dimensional, open-source, image-correlation program Ncorr. Included in the design with the die and punch was a support for a camera, lighting, and the associated optical components. The equipment was designed to be mounted on an Instron 3382, $100 \mathrm{kN}$ capacity, universal testing machine. A universal testing machine is easily controlled in relation to a shop-floor forming press, and loads and displacements can be recorded.

\section{DESIGN}

The equipment design consists of two parts: the punch, the die, and the support that loads the test piece and the optical system for photographing the specimen during deformation. The assembly of these two parts to form the complete system is shown in Fig. 2. Similar equipment for conducting the MK experiment and photographing the sample in situ was presented by Brunet, Mguil, and Morestin ${ }^{16}$ and Brunet and Morestin ${ }^{17}$ although in the case of their experiment, the punch and camera are fixed and the die moves over the punch. Here, it is the opposite.

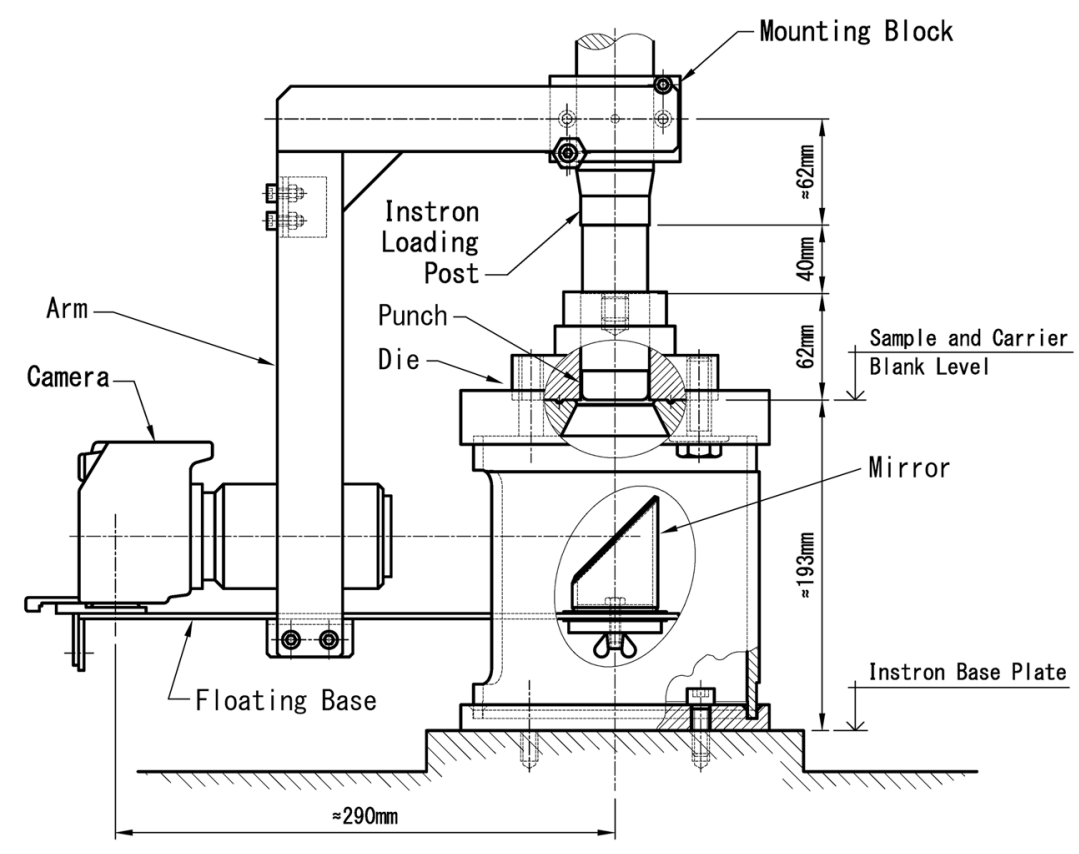

FIG. 2. Assembly drawing of the punch, die support, and optical system. 


\section{A. Punch, die, and support}

For this MK test, the sample and its corresponding carrier blank are fixed in a two-part die, shown near the top of Fig. 2. The die was fabricated from SAE 1045 steel that was quenched and tempered to a hardness of about 45 Rockwell C. A slight recess $0.5 \mathrm{~mm}$ deep and $80 \mathrm{~mm}$ in diameter was cut into the lower part of the die to center the sample and carrier blank. Within this reduced section, there is a $65 \mathrm{~mm}$ diameter draw bead to fix the sample and carrier blank. An impression of the draw bead is made in the specimens before the test, and this serves for alignment when the sample and carrier blank are mounted for the experiment. The die is held together with eight $14.29 \mathrm{~mm}$ standard-thread bolts evenly spaced on a $100 \mathrm{~mm}$ bolt circle. The lower die piece has a tapered hole with a minimum diameter of $45 \mathrm{~mm}$ to provide clearance for deformation of the test pieces. The edge of this clearance hole is radiused to prevent specimen tearing. The upper part of the die has a precision machined $40 \mathrm{~mm}$ diameter hole to act as a guide and sleeve for the punch. We machined the punch from 70:30 brass to facilitate making changes to the punch radius, settling on $4 \mathrm{~mm}$. These dimensions all scale to those given in the norm ISO 12004-2. ${ }^{6}$ The die assembly sits on a $168.3 \mathrm{~mm}$ diameter seamless tube that is $165 \mathrm{~mm}$ long. The standard tube thickness of $7.11 \mathrm{~mm}$ is more than sufficient to support the $100 \mathrm{kN}$ load capacity of the testing frame. A rectangular $90 \mathrm{~mm} \times 120 \mathrm{~mm}$ relief hole was cut into the side of the tube to permit the entry of the optical system. The entire die assembly and tube sit on a plate bolted to the lower base of the Instron universal testing machine.

\section{B. Optical system}

Because the MK test-section deformation is planar, a single camera and two-dimensional image-correlation program can be used to record the evolution of deformation. The single requirement is that the distance between the camera and the sample surface is maintained constant during the entire experiment. This assures a constant focus and magnification. Thus, the optical system consists of a floating base to which a mirror and the camera are mounted. This base is attached through arms to the testing machine crosshead. Thus, the floating base lowers the exact amount as the crosshead and the stretching specimen surface.

A mounting block was attached through a rod to the machine's crosshead. Pins and small bolts machined eccentrically were inserted into the mounting block. The arms, being held by the pins and eccentric bolts, then slip fit around the mounting block. By rotating the eccentric bolts, the pitch and roll of the arms, and hence the camera and mirror, can be finely adjusted to center the specimen's surface in the camera's field of view. The mounting block is shown enlarged in Fig. 3. Such an assembly permits the optical system to return to an exact position when it is slid in and out before and after testing. This arrangement allowed the experiment to be halted at an intermediate strain, with the optical system and die removed and the carrier blank given a recrystallizing heat treatment. After the thermal treatment, all components could then be remounted in their exact previous positions and the specimen loading and image acquisition resumed.

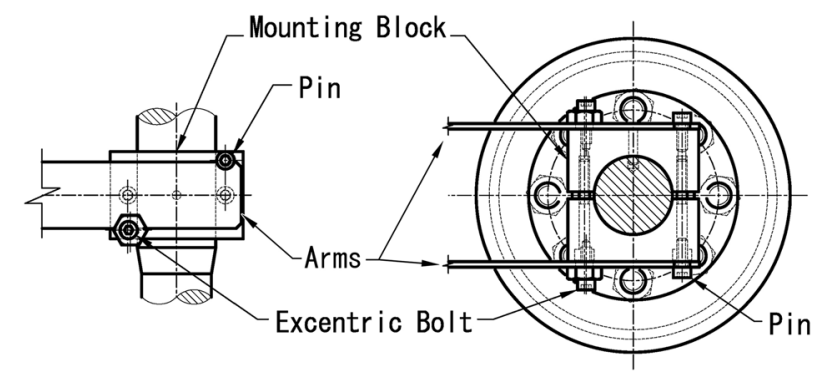

FIG. 3. An isolated side and plan view of the optical-system mounting block.

We used an elliptical, front-surface, optical-quality mirror-mounted at $45^{\circ}$ with a commercial support to a slot cut in the floating base-to make the specimen's deforming surface visible to the camera. For these forming-limit experiments, high image resolution rather than high recording speed is advantageous. Thus, a commercial photographic camera, Nikon D3300 with a $4500 \times 3000$ pixel resolution CCD, was chosen rather than a high-speed video camera. An AF-S DX Micro NIKKOR $85 \mathrm{~mm}$ lens allowed the camera to be focused over the short distances associated with this experiment. We mounted the camera to the base with a wedge-plate monopod

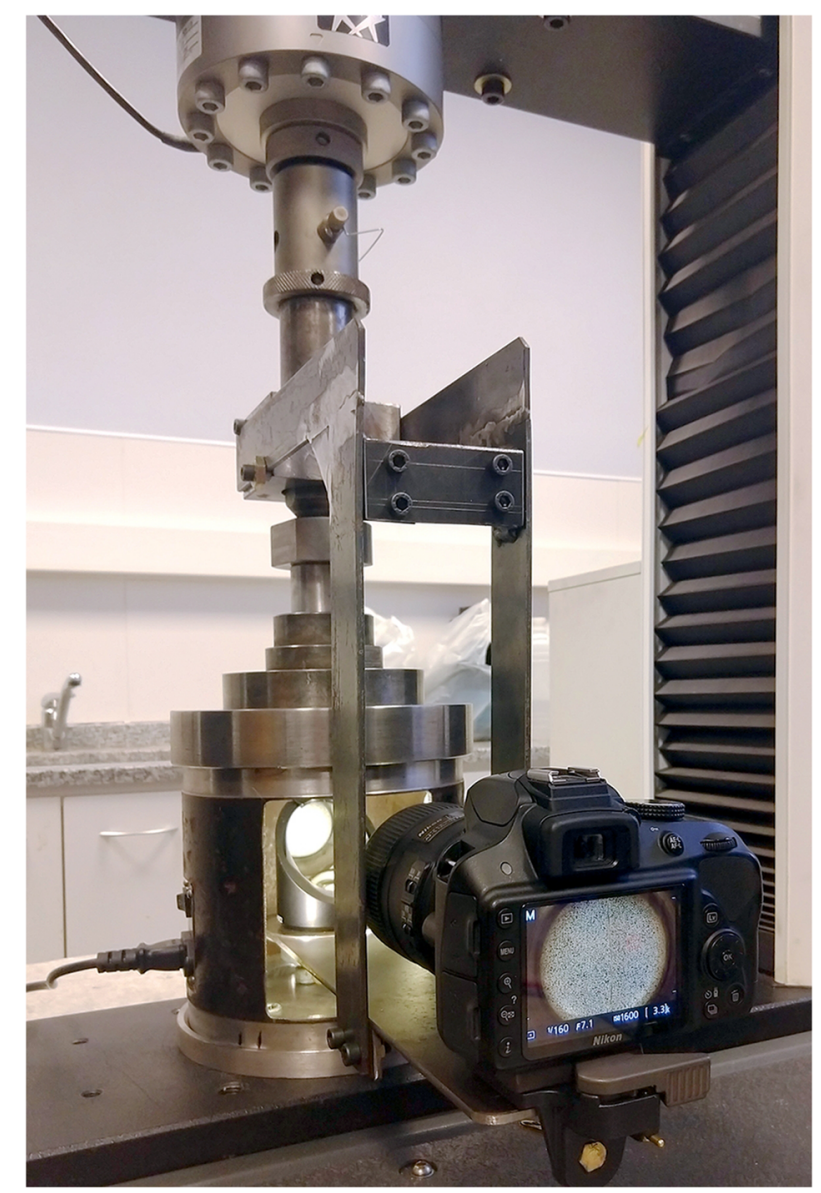

FIG. 4. A view of the assembled device after testing the $52 \mathrm{~mm}$-wide hourglass sample. The mirror and its support are seen attached to the floating base. The specimen is illuminated by LED strips mounted on either side of the support-tube's aperture. Light from the LEDs reflects off the mirror upwards to the specimen surface. The deformed sample's speckle pattern is seen on the camera's screen. 
mount. In this way, the camera could also be simply removed and remounted in the exact same position. During the experiment, with the camera's mirror in the retracted position, a Shoot MC-DC2 timer remote triggered the camera's shutter at $1 \mathrm{~s}$ intervals.

Finally, light-emitting diode (LED) strips, mounted internally on either side of the support tube, provide adequate and uniform illumination of the sample throughout testing.

The entire assembled and operating device is shown in Fig. 4, after the testing of a $52 \mathrm{~mm}$-wide, hourglass specimen.

\section{EXPERIMENTAL IMPLEMENTATION}

When implementing MK experiments with this instrument, several considerations must be made concerning sample geometries, materials, friction and lubrication, painting the speckle pattern, and stopping the experiment at intermediate deformations, in order to recrystallize the carrier blank.

\section{A. Sample geometries}

As discussed in the norm, various geometries of samples and carrier blanks can be used. By implementing each of these designs, all of the strain states between uniaxial and equibiaxial tension can be obtained. Four geometries are noteworthy, and shapes and dimensions from actual experiments are shown in Figs. 5(a)-5(d). For use with the current

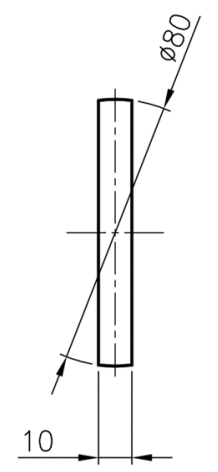

(a)

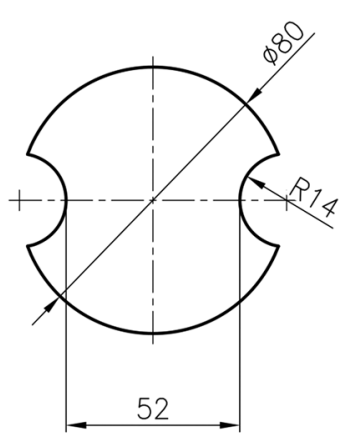

(c)

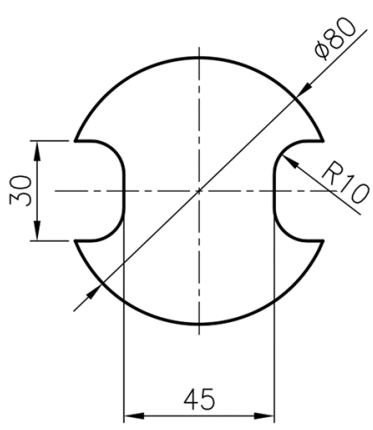

(b)

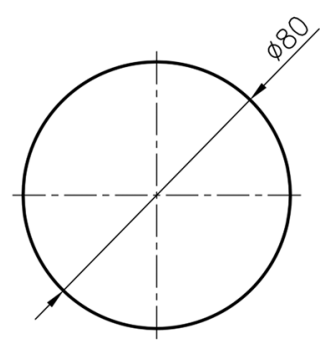

(d)
FIG. 5. The four noteworthy specimen geometries used with this equipment: (a) flat parallel sides (approximates uniaxial tension), (b) parallel sides with an enlarged gripping area (dog bone), (c) radiused to a minimum width in the center (hourglass), and (d) a full-diameter disk (equibiaxial tension). equipment, all samples were $80 \mathrm{~mm}$ in length/diameter. The four geometries are flat-parallel sides (approximates uniaxial tension) [Fig. 5(a)], parallel sides with an enlarged gripping area (dog bone) [Fig. 5(b)], radiused to a minimum width in the center (hourglass) [Fig. 5(c)], and a full-diameter disk (equibiaxial tension) [Fig. 5(d)]. Flat samples with parallel sides minimize constraint across the specimen width and typically give data points between uniaxial tension and plane-strain deformation. The dog-bone samples have enlarged ends to prevent tearing in the case of high-strength materials. These ends also constrain contraction of the specimen width, and the samples are most useful for obtaining deformation states near plane strain. The hourglass sample shape offers even more constraint, and it is useful for the full right-hand side of the FLD. A full disk gives only equibiaxial strain fields.

For the current experiments, the samples and carrier blanks were laser cut. The central holes of the carrier blanks were rectified using a lathe and grinding stone spinning at high speed in a Dremel tool. Finally, the laser cut surfaces were polished with diamond jeweler's files and then $\mathrm{SiC}$ paper with grits between 120 and 320 .

\section{B. Materials}

Samples are obviously cut from the material of interest. The norm ISO $12004-2^{6}$ states that the carrier blank must be from a sheet at least as thick as the sample and should have the same or greater capacity for deformation. Because of its good formability, moderate strain-rate sensitivity, and hardening capacity, we selected a drawing-quality steel sheet for the carrier-blank material. In addition, we performed our experiments in two stages with an intermediate recrystallization of the carrier blank.

\section{Friction and lubrication}

In the MK experiment, high friction and good lubrication are needed at different interfaces. A well lubricated surface is needed between the punch and the sample carrier blank. For this, we used the aerosol, solid-lubricant Molykote $321 \mathrm{R}$.

On the other hand, it is necessary to have high friction between the carrier blank and the sample to transmit forces. In this case, the norm suggests sand blasting the interior surfaces of the sample and carrier blank. This was done for our experiments with this equipment.

\section{Speckle pattern}

The speckle pattern is typically painted with aerosol cans, first a solid white background and then an overpaint of fine black spots. This painted layer must deform equally to the sample without pealing or flaking from the sample's surface. We have achieved the greatest success with a slow drying white base paint, waiting only as long as necessary for application of a second coat (in our case $1 \mathrm{~h}$ ) to apply the black speckles. A fast drying paint, $10 \mathrm{~min}$, was used for the speckles.

\section{E. Intermediate heat treatment of the carrier blank}

When performing MK experiments, it is not uncommon to experience cracking at the surface of the carrier blank's 
central hole. Although a drawing-quality steel carrier blank has excellent ductility, its capacity to deform is often insufficient. A two-stage approach to the forming limit experiment overcomes this problem. With the control afforded by loading in a universal testing machine, the MK test can be halted at an intermediate strain and disassembled. The carrier blanks can then be given a recrystallizing heat treatment in a furnace with an inert atmosphere. Owing to the smaller sample size, it was possible to perform this heat treatment within a chamber that was slid into a conventional muffle furnace. The chamber, which allowed passage of argon gas, was sufficiently sealed to the atmosphere to prevent oxidation of the carrier blanks. A 15 min heat treatment at $850{ }^{\circ} \mathrm{C}$ followed by an ambient cooling fully recrystallized the carrier blank. Cracks never appeared after using this procedure.

\section{RESULTS}

Samples of a $0.8 \mathrm{~mm}$ thick, Zn-0.09Cu-0.04Ti wt. \% sheet (designated by the manufacturer as $\mathrm{Zn} 20$ ) and a $0.9 \mathrm{~mm}-$ thick drawing-quality steel carrier blank were tested with the new device. Results spanning the entire FLD from uniaxial to balanced-biaxial tension were obtained. Figure 5 shows four noteworthy geometries with their widths drawn to scale. In these and all other cases, the samples' vertical axes were perpendicular to the prior rolling direction, and the necking instability develops across the specimen width, parallel to the rolling direction. The limit-strain results, calculated using the Bragard ${ }^{10}$ concept, cover the entire range of the FLD, as shown in Fig. 6. Each of these points is taken from a fitted curve passing through the point of maximum deformation and corresponds to a single experiment. They lie in a bowl-shaped profile typical of FLDs and are consistent with previous results for the Zn20 sheet obtained by Schlosser and Signorelli. ${ }^{18}$ It is notable that Schlosser and Signorelli's data were taken using Nakazima's technique, ${ }^{7}$ a $40 \mathrm{~mm}$ punch and circle-grid measurements. This is a technique completely distinct from the current MK test. These authors present their results as either homogeneous (secure) deformation or necked (insecure) deformation. Data points from multiple circle measurements

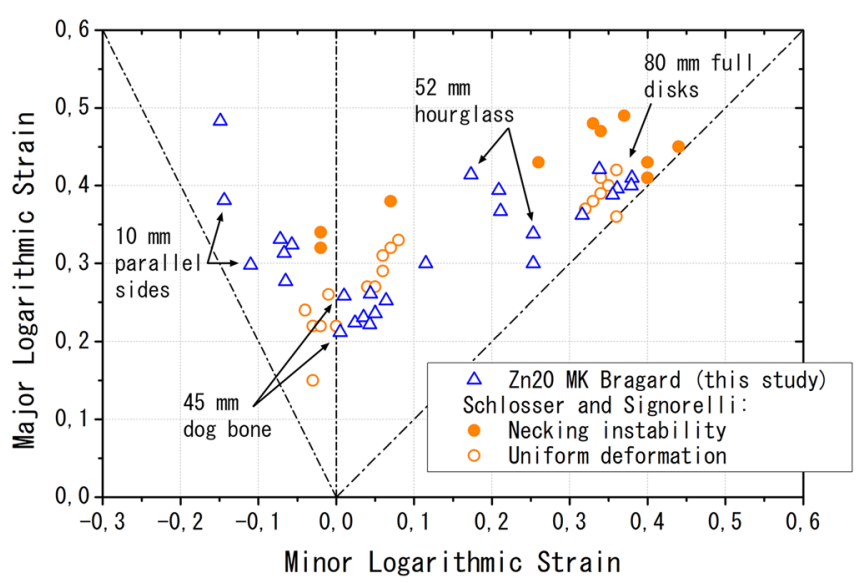

FIG. 6. Current MK data for $\mathrm{Zn} 20$ ( $\mathrm{Zn}-\mathrm{Cu}-\mathrm{Ti}$ ) compared to those from the previous work by Schlosser and Signorelli. ${ }^{18}$

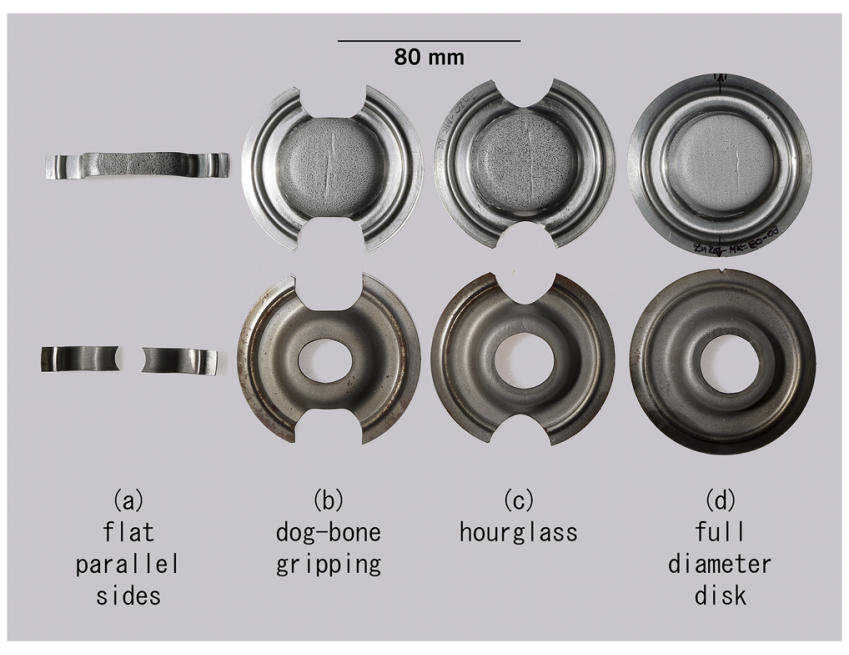

FIG. 7. Deformed samples and their associated carrier blanks corresponding to the four noteworthy geometries shown in Fig. 5.

typically result from a single experiment. Raghavan ${ }^{19}$ demonstrated for drawing-quality and interstitial-free steels that the MK and Nakazima geometries should yield equivalent results. The coincidence of these data verifies the results from the new device and image correlation analysis. Raghavan's results also showed that FLD measurements are sensitive to sheet thickness. Schlosser and Signorelli's material was slightly thinner, $0.65 \mathrm{~mm}$, than the material used in this study. However, this difference appears to have had a minimal effect.

Figure 7 shows four deformed samples corresponding to the geometries shown in Fig. 5 and their associated carrier blanks. The samples were tested past the point of instability to fracture. It is clear that the draw bead held each sample/carrier blank securely and that the samples experienced only stretching. An instability and crack run across the width of the samples at the point of minimum width-with the exception of the parallel-sided sample that approximates uniaxial tension. The hole in the carrier blank has expanded dramatically yet there is no cracking at either the central hole or radiused surfaces. Data from the center of the sample will thus provide valid limit strains. The other samples and carrier blanks behaved similarly. It should be noted that in the case of the $10 \mathrm{~mm}$ parallel-sided test, the carrier blank was split into individual pieces before testing, and no recrystallization treatment was required. The locations of the data from these samples are indicated in Fig. 6. It can be seen that these points cover the full span of the FLD. Intermediate points were obtained by varying the dimensions of these four sample types.

Taking a horizontal strain profile through the center of the samples with Ncorr, using an image just before fracture, allows the Bragard criterion to be applied. Typical results for the $52 \mathrm{~mm}$ wide sample are shown in Fig. 8. Obviously, deformation is very uniform in the central region of the sample, with the exception of the instability. This zone of high strain is discarded for the Bragard analysis. The ISO $12004-2^{6}$ norm specifies that the inner boundary for fitting be defined by the point where the second derivative of the major true (logarithmic) strain is a maximum. This border is seen in Fig. 8. The fitted points are highlighted, and the second derivative appears in the bottom most plot. For a miniature size sample, the norm 

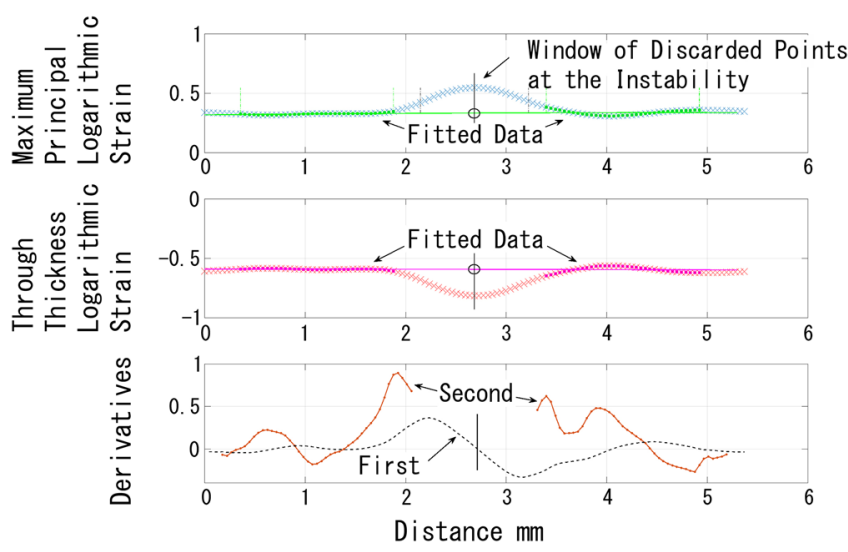

FIG. 8. Measured strain profiles from the $52 \mathrm{~mm}$ sample and the Bragard fit from the image taken immediately before the first appearance of cracking.

is not applicable in specifying the outer boundaries for fitting. However, because the deformation profile is so flat, the specific outer boundary taken will have little effect on the results. Again, the other samples' behaviors were equivalent. Finally, the values of the fitted lines at the center of the necked region specify the FLD values. The norm stipulates that $\varepsilon_{2}$, the minor FLD strain, be calculated from the through-thickness strain $\left(\varepsilon_{3}\right)$ using constancy of volume. The major strain, $\varepsilon_{1}$, comes directly from the fitting.

As yet, we have not decided on a time-history method for obtaining limit strains. However, for the $52 \mathrm{~mm}$ wide specimen, Fig. 9 shows the evolution of Lagrangian strain fields perpendicular and parallel to the instability during the second stage of sample deformation, after the recrystallization of the carrier blank. The times at which the images were recorded

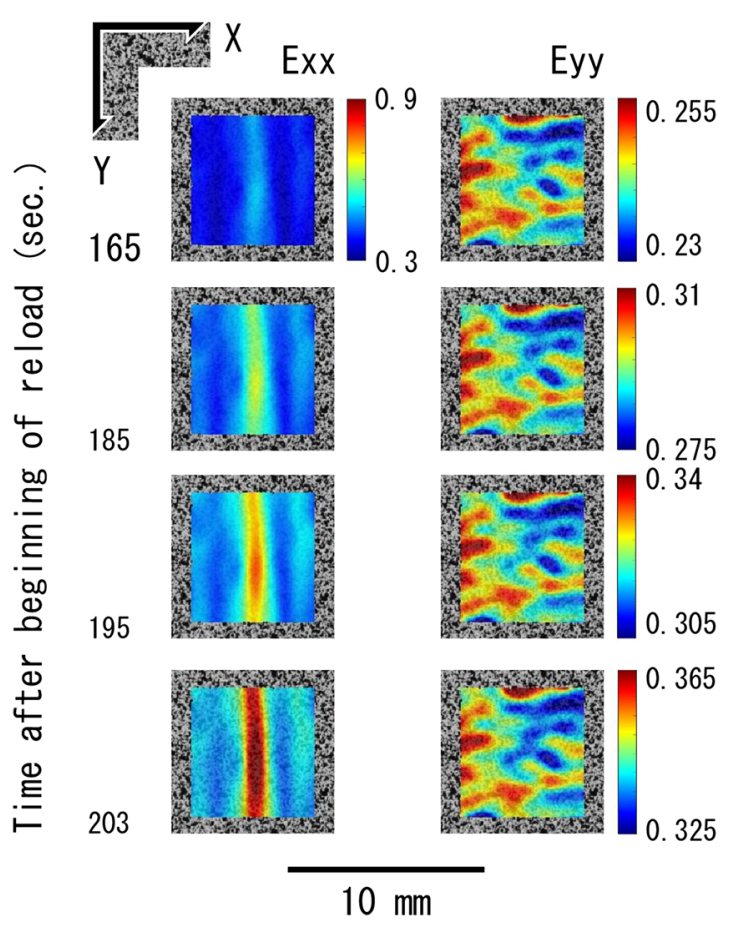

FIG. 9. A sequence of strain fields parallel and perpendicular to the necking instability for the $52 \mathrm{~mm}$ sample showing the instability's development in time. The image at $203 \mathrm{~s}$ was the last before fracture was observed. One image was recorded every second. are indicated. The strains perpendicular to the neck are plotted to an absolute scale to show how the instability develops. The strains parallel to the instability are plotted to relative scales. These parallel strains show no indication of the developing instability, and their pattern is constant, as one would expect. The camera records sharp images as the deformation proceeds, and strains are calculated without a problem. Zones where the deformation is beginning to localize are evident, particularly in the final images close to the instability. These images will clearly provide data for calculation of time-dependent limit strains.

\section{DISCUSSION AND CONCLUSIONS}

We built a laboratory-scale device to measure forming limit curves based on the MK sample geometry. The equipment takes advantage of recently developed experimental techniques: digital image correlation, the open-source program Ncorr, LED lighting, and high-resolution and high-frequency digital photography, a $4500 \times 3000$ pixel image every second. Because we used a laboratory-scale universal testing machine to load the specimens, we had precise control of sample deformation. Thus, we were able to stop the test at intermediate deformations to recrystallize the steel, MK carrier blank. Because of this treatment, the carrier blanks never suffered cracks at the central-hole edge, which would have invalidated the experiment.

The four geometries used in this initial study (parallel sides, dog bone, hourglass, and full disk) cover the entire FLD field. With laser cutting, the widths of the samples are very precise, and through slight variations, any strain path can be followed.

Our results were validated through comparison to previous experiments conducted using a completely independent FLD specimen geometry and strain-measurement technique on the same material.

The MK test is strictly a plane-stress experiment because there is no contact between the punch and the sample, as occurs in the Nakazima hemispherical test. The sample is loaded by friction with the carrier blank, particularly in the area of the punch radius. Because of the hole in the carrier blank, there is no friction in the area where the instability develops. Historically, the MK test has not been very popular because it was difficult to arrive at a combination of geometries in the punch and matrix that permit sufficient sample deformation without fracture at the edge of the carrier-blank hole. We overcame these limitations by adhering to dimensional proportions outlined in the ISO $12004-2^{6}$ norm and with the carrier-blank recrystallization treatment.

Finally, a miniature sample size and universal testing machine loading offer advantages and potentials. They permitted halting the test to recrystallize the carrier blank and, second, allowed a number of experiments to be performed with a limited quantity of material. This can be important for the university or research environment. It might also be possible to eventually investigate changes in loading path through multiple MK experiments, first a conventional-sized test to preload the material and then an FLD determination with the miniature device. 


\section{ACKNOWLEDGMENTS}

This work was supported by CONICET (Consejo Nacional de Investigaciones Científicas y Técnicas, Argentina) through Grant Nos. PICT 2013-0558 and ECOS-SUD A16E03. The authors also wish to thank Dr. Mariano Serenelli for his work in acquiring the data presented by Schlosser and Signorelli. ${ }^{18}$

${ }^{1}$ D. Banabic, Sheet Metal Forming Processes (Springer-Verlag, Berlin, 2010) ${ }^{2}$ W. Emmens, Formability: A Review of Parameters and Processes that Control, Limit or Enhance the Formability of Sheet Metal (Springer-Verlag, Berlin, 2011).

${ }^{3}$ S. P. Keeler and W. A. Backofen, ASM Trans. Q. 56, 25 (1963).

${ }^{4}$ G. M. Goodwin, SAE Technical Paper No. 680093 (1968), p. 380.

${ }^{5}$ M. Gensamer, Trans. Am. Soc. Met. 36, 30 (1946); Metallogr. Microstruct. Anal. 6, 171 (2017).

${ }^{6}$ ISO 12004-2:2008, Metallic Materials-Sheet and Strip-Determination of Forming-Limit Curves. Part 2: Determination of Forming-Limit Curves in the Laboratory (2008).
${ }^{7}$ K. Nakazima, T. Kikuma, and K. Hasuka, Yawata Technical Report No. 264 (1968), p. 8517.

${ }^{8}$ S. Hecker, Sheet Met. Ind. 52, 671 (1975).

${ }^{9}$ Z. Marciniak and K. Kuczynski, Int. J. Mech. Sci. 9, 609 (1967).

${ }^{10}$ A. Bragard, J. C. Baret, and H. Bonnarens, Rapport Centre de Recherche de la Metallurgie No. 33 (1972), p. 53.

${ }^{11}$ M. A. Sutton, J.-J. Orteu, and H. W. Schreier, Image Correlation for Shape, Motion and Deformation Measurements (Springer, New York, 2009).

${ }^{12}$ P. Vacher, A. Haddad, and R. Arrieux, CIRP Annals-Manuf. Technol. 48, 227 (1999).

${ }^{13}$ See https://www.gom.com/metrology-systems/aramis.html for information about the GOM commercial digital image correlation code.

${ }^{14}$ J. Blaber, B. Adair, and A. Antoniou, Exp. Mech. 55, 1105 (2015).

${ }^{15}$ W. Hotz, M. Merklein, A. Kuppert, H. Friebe, and M. Klein, Key Eng. Mater. 549, 397 (2013)

${ }^{16}$ M. Brunet, S. Mguil, and F. Morestin, J. Mater. Process. Technol. 80-81, 40 (1998).

${ }^{17}$ M. Brunet and F. Morestin, J. Mater. Process. Technol. 112, 214 (2001).

${ }^{18}$ F. Schlosser and J. Signorelli, Mec. Comput. 33, 1271 (2014), https:// cimec.org.ar/ojs/index.php/mc/article/view/4724/4656.

${ }^{19}$ K. S. Raghavan, Metall. Trans. A 26A, 2075 (1995). 